\title{
P04-54 LB. Genetic signature analysis identifies the CD4i region of gp I 20 as a determinant of broadly NAb responses in HIV-I-infected individuals
}

\author{
G Gnanakaran*1, T Bhattacharya1 ${ }^{1}$ M Daniels ${ }^{1}$, A Lapedes1 ${ }^{1}$ F Gao ${ }^{2}$,

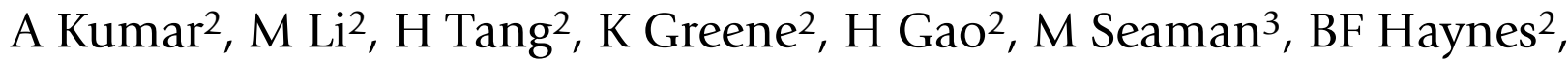 \\ DC Montefiori² and BT Korber ${ }^{1}$
}

Address: ${ }^{1}$ Los Alamos National Labs, Los Alamos, USA, ${ }^{2}$ Duke University, Durham, USA and ${ }^{3}$ Harvard Medical School, Boston, USA

* Corresponding author

from AIDS Vaccine 2009

Paris, France. 19-22 October 2009

Published: 22 October 2009

Retrovirology 2009, 6(Suppl 3):P394 doi:10.1186/1742-4690-6-S3-P394

This abstract is available from: http://www.retrovirology.com/content/6/S3/P394

(c) 2009 Gnanakaran et al; licensee BioMed Central Ltd.

\section{Background}

Structure-assisted immunogen design plays a central role in current efforts to create an effective HIV-1 vaccine. Much of this effort utilizes available structural information on Env as an antigen. Considerably less is known about the molecular determinants of Env immunogenicity. We sought to close this gap by identifying amino acid signatures in serum-derived gp160 genes that associate with broadly neutralizing antibody responses.

\section{Methods}

Computational tools for robust clustering of like-patterns of neutralization potency were designed based on Kmeans clustering, factoring in the uncertainty that results from limited sampling (bootstrap) and assay variability (noise). Phylogenetically corrected methods were used to identify associations between genetic mutations and distinct neutralization clusters.

\section{Results}

For validation, we first analyzed Env sequences from 251 clonal viruses that were differentially sensitive to $\mathrm{MAb}$ b12. Three amino acid positions that are known to occur in the b12 binding surface of gp120 strongly associated with b12 potency. An additional three positions in gp120 and one in the C-heptad repeat of gp41 were identified that could be distal sites that impact b12 potency. Next, we analyzed a checkerboard-style neutralization dataset comprising a multi-subtype panel of clonal Env-pseudotyped viruses $(n=25)$ and sera from 68 infected individuals from whom a serum gp 160 sequence was derived by single genome amplification (SGA). Three distinct clusters of sera with high, medium and low neutralization potencies were identified. By analysis of the SGA-derived Env sequences from these individuals, three amino acid positions were identified in the CD4-inducible (CD4i) region of gp120 that strongly associated with neutralization potency.

\section{Conclusion}

Our findings suggest that the CD4i region of gp120 is a key determinant (although not necessarily a target) of broadly NAb responses in HIV-1-infected individuals. In addition to providing new insights for immunogen design, these computational tools may be useful in delineating the neutralization epitopes of novel monoclonal antibodies. 